\title{
Overview of quarkonium production in heavy-ion collisions at LHC
}

\author{
Byungsik Hong ${ }^{1, a}$ on behalf of the ALICE, ATLAS and CMS Collaborations \\ ${ }^{1}$ Department of Physics, Korea University, Seoul 02841, Republic of Korea
}

\begin{abstract}
Quarkonium has been regarded as one of the golden probes to identify the phase transition from confined hadronic matter to the deconfined quark-gluon plasma (QGP) in heavy-ion collisions. Recent data on the yields and momentum distributions of $J / \psi$ and $\Upsilon$ families in $\mathrm{pp}, \mathrm{pPb}$, and $\mathrm{PbPb}$ collisions at the Large Hadron Collider (LHC) are reviewed. The possible implications related to the propagation of quarkonia in the deconfined hot, dense matter and the modified parton distribution function (PDF) in cold nuclei are also discussed.
\end{abstract}

\section{Introduction}

Several observables have been proposed to identify and study the detailed characteristics of the phase transition from the confined hadronic matter to the deconfined QGP. The production of various $c \bar{c}$ and $b \bar{b}$ bound states is one of them. The heavy quarkonia are generated in early stage of the collision due to their large masses and expected to be sequentially resolved by color Debye screening at different temperatures, depending on their binding energies, during evolution [1]. Therefore, the data on the yields and momentum spectra of quarkonia may provide invaluable information on the medium temperature and the characteristics of quantum chromodynamics (QCD) in high density and temperature environment [2].

Another production mechanism is the random recombination (or regeneration) of heavy quarks and their antiquarks [3]. Contrary to the color Debye screening effect, the regeneration process enhances the quarkonium yields, especially, at the low-transverse momentum $\left(p_{T}\right)$ range. Recently, various transport models have incorporated with not only color Debye screening, but also the regeneration effects to reproduce the experimental data $[4,5]$. As a result, the quarkonium data are expected to constrain probabilities of the two competing processes.

More recently, the quarkonium production in asymmetric $\mathrm{pPb}$ collisions became available at LHC. The quarkonium data in $\mathrm{pPb}$ are useful to recognize and quantify the initial-state effects, such as nuclear modification of PDF, energy loss in cold nuclear matter, and $p_{T}$ broadening due to multiple scattering [6]. These initial-state nuclear effects need to be taken into account to isolate any genuin signals caused by the high-density QCD medium in heavy-ion collisions. In the meanwhile the quarkonium data in pp are important for heavy-ion physics as they serve as important references. Furthermore, the production mechanism of quarkonia in $\mathrm{pp}$ is yet to be understood.

ae-mail: bhong@korea.ac.kr 

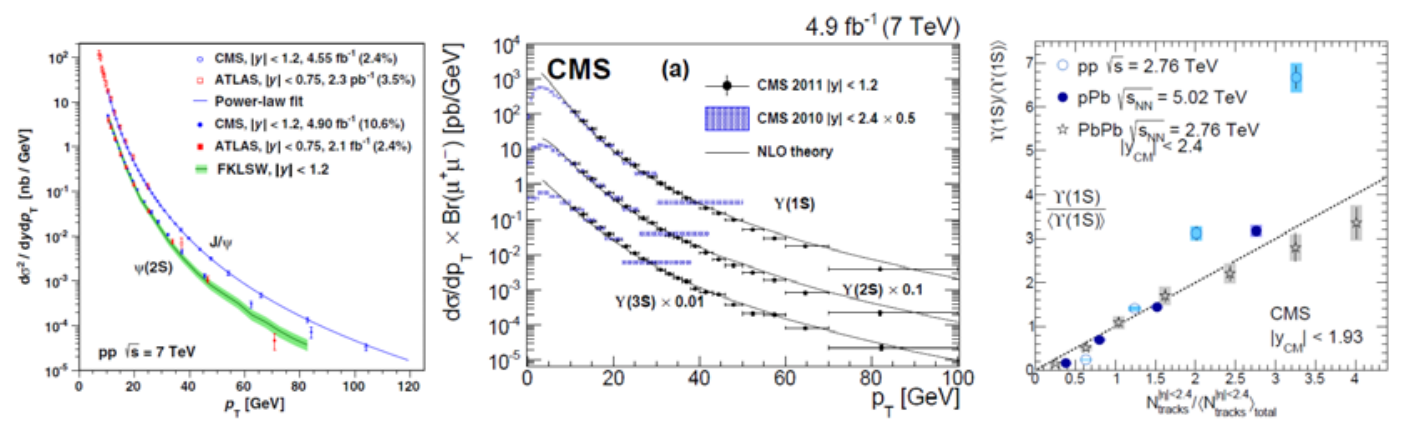

Figure 1. (Left) Differential $p_{T}$ cross sections from CMS $(|y|<1.2)$ [7] and ATLAS $(|y|<0.75)[8,9]$ for $J / \psi$ and $\psi(2 \mathrm{~S})$ in pp at $7 \mathrm{TeV}$. The error bars show the statistical and systematic uncertainties added in quadrature. The uncertainties from integrated luminosities and branching fractions are given by the percentages in the legend. The curve is a fit to the $J / \psi$ cross section from CMS by using a power-law function, and the band labeled by FKLSW shows a cross-section calculation from NRQCD+NLO for $\psi(2 S)$ [11]. (Center) Differential $p_{T}$ cross sections times dimuon branching fractions for $\Upsilon(n S)$ within $|y|<1.2$ in pp at $7 \mathrm{TeV}$ from CMS [12]. The $\Upsilon(2 S)$ and $\Upsilon(3 S)$ data are scaled by 0.1 and 0.01 , respectively, for clearer display. The error bars show the total uncertainty, excluding the systematic uncertainty in the integrated luminosity, and the horizontal bars show the bin widths. Previous CMS measurements for $|y|<2.4$ are shown by the cross-hatched areas [13]. The solid lines are the NRQCD+NLO calculations [14]. (Right) The $\Upsilon(1 \mathrm{~S})$ cross sections as a function of charged-track multiplicity measured in $|\eta|<2.4$ in $\mathrm{pp}$ and $\mathrm{PbPb}$ at $2.76 \mathrm{TeV}$ and $\mathrm{pPb}$ at $5.02 \mathrm{TeV}$ [15]. Cross sections and $N_{\text {tracks }}^{|\eta|<2.4}$ values are normalized by their corresponding activity-integrated values. The dotted line is a linear function with a slope of unity. The error bars indicate the statistical uncertainties, and the boxes represent the point-to-point systematic uncertainties.

This paper summarizes some of recent experimental data on the $J / \psi$ and $\Upsilon$ productions in pp, pPb, and $\mathrm{PbPb}$ collisions at $\mathrm{LHC}$. The implications of the data for the production mechanism, the initialand final-state effects are also discussed.

\section{Quarkonium production in pp}

The left panel of Fig. 1 shows the double-differential cross sections of promptly produced $J / \psi$ and $\psi(2 \mathrm{~S})$ in pp at $\sqrt{s}=7 \mathrm{TeV}$ from ATLAS and CMS [7-9]. The curve represents a power-law fit function to the $J / \psi$ cross section [10], whereas the band labeled by FKLSW represents the global fit to the $\psi(2 \mathrm{~S})$ cross section by the perturbative QCD (pQCD) at the next-to-leading order (NLO) level [11]. The experimental $\psi(2 \mathrm{~S})$ cross section are well described by the NLO pQCD calculations.

The $\Upsilon(n S)$ differential cross sections, multiplied by dimuon branching fractions, are plotted as a function of $p_{T}$ in the middle panel of Fig. 1 over the rapidity range $|y|<1.2$ in pp at $7 \mathrm{TeV}$ [12]. They are compared with the earlier CMS data [13], scaled by 0.5 to account for the smaller rapidity coverage in the latest data. Previously, the NLO analysis of non-relativistic QCD (NRQCD) successfully described the $\Upsilon(n S)$ spectra for $p_{T}<50 \mathrm{GeV}$. The extended calculations to $p_{T}=100 \mathrm{GeV}$ [14], shown by solid lines in the middle panel of Fig. 1, agree with the recent CMS data. The new data with an extended $p_{T}$ reach should be useful in evaluating long-distance matrix element (LDME) and studying the $p_{T}$-dependent corrections in NRQCD and other models.

One of the most interesting features in the quarkonium production in pp is shown in the right panel of Fig. 1, which compares the $\Upsilon(1 \mathrm{~S})$ cross section vs. charged-track multiplicity measured in $|\eta|<2.4$, 

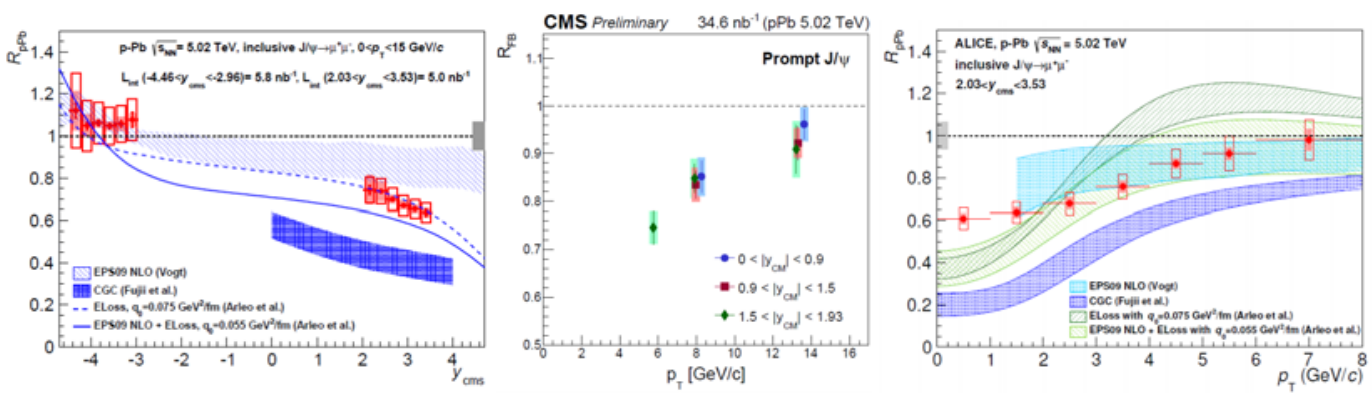

Figure 2. (Left) Nuclear modification factor $R_{p P b}$ of inclusive $J / \psi$ as a function of the center-of-mass rapidity in $\mathrm{pPb}$ at $5.02 \mathrm{TeV}$ from ALICE [18]. The error bars represent the statistical uncertainties. The open and the shaded boxes correspond to the uncorrelated and the partially correlated, respectively, systematic uncertainties. The full boxes around $R_{p P b}=1$ show the the correlated uncertainties. Various model calculations are also shown. (see the text for details.) (Center) Forward-to-backward yield ratio $R_{F B}$ of prompt $J / \psi$ in three different rapidity ranges as a function of $p_{T}$ in $\mathrm{pPb}$ at $5.02 \mathrm{TeV}$ from CMS [22]. (Right) $R_{p P b}$ of inclusive $J / \psi$ as a function of $p_{T}$ at forward rapidities in $\mathrm{pPb}$ at $5.02 \mathrm{TeV}$ from ALICE [23]. Various model calculations are also displayed. (see the text for details.)

$N_{\text {tracks }}^{|\eta|<2.4}$, in $\mathrm{pp}, \mathrm{pPb}$, and $\mathrm{PbPb}$ [15]. Note that the cross sections and $N_{\text {tracks }}^{|\eta|<2.4}$ values are normalized by their corresponding activity-integrated values. The self-normalized cross section ratios increase with event multiplicity: the $\mathrm{pPb}$ and $\mathrm{PbPb}$ data increase almost linearly with $N_{\text {tracks }}^{|\eta|<2.4}$, but the pp data show much stronger positive correlation between the $\Upsilon$ yield and the underlying event activity. A similar trend can be also observed in the $J / \psi$ production at $7 \mathrm{TeV}$ [16]. A possible interpretation would be the multiple parton-parton interactions in a single pp collision [17].

\section{Quarkonium production in $\mathrm{pPb}$}

Figure 2 shows the center-of-mass rapidity and $p_{T}$ dependences of the $J / \psi$ yields in $\mathrm{pPb}$ at 5.02 $\mathrm{TeV}$. The left panel of Fig. 2 from ALICE shows that the inclusive $J / \psi$ yield in $\mathrm{pPb}$ is suppressed with respect to the one in binary-scaled pp collisions at the forward (p-going) rapidities, whereas it is unchanged at the backward (Pb-going) rapidities [18]. The data are compared with the calculations by a NLO color-evaporation-model (CEM) using the EPS09 nPDF parameterization [19] and a coherent parton-energy-loss model with and without EPS09 shadowing effect [20]. The calculation in the color-glass condensate (CGC) framework [21] is also displayed. Within the uncertainties, the models based on shadowing and the coherent energy loss are able to describe the data, but the CGC-based calculation overestimates the suppression. Note that none of the above mentioned models include a suppression due to the break-up of the $c \bar{c}$ pairs.

CMS analyzed the prompt $J / \psi$ and non-prompt $J / \psi$ from $B$ decay, separately, using the precise measurement of the decay length. For example, the middle panel of Fig. 2 shows the forward-tobackward yield ratios $R_{F B}$ of prompt $J / \psi$ in three different rapidity bins as a functions of $p_{T}$ [22]. The $R_{F B}$ distributions decrease monotonically towards smaller $p_{T}$ without any strong rapidity dependence. The prompt (and also non-prompt, not shown here) $J / \psi$ yields at forward rapidities are smaller than those at backward rapidities, which is consistent with the ALICE data.

The right panel of Fig. 2 displays the $R_{p P b}$ distribution of inclusive $J / \psi$ as a function of $p_{T}$ at forward rapidity region where the suppression is largest [23]. The data are compared to the calculations 

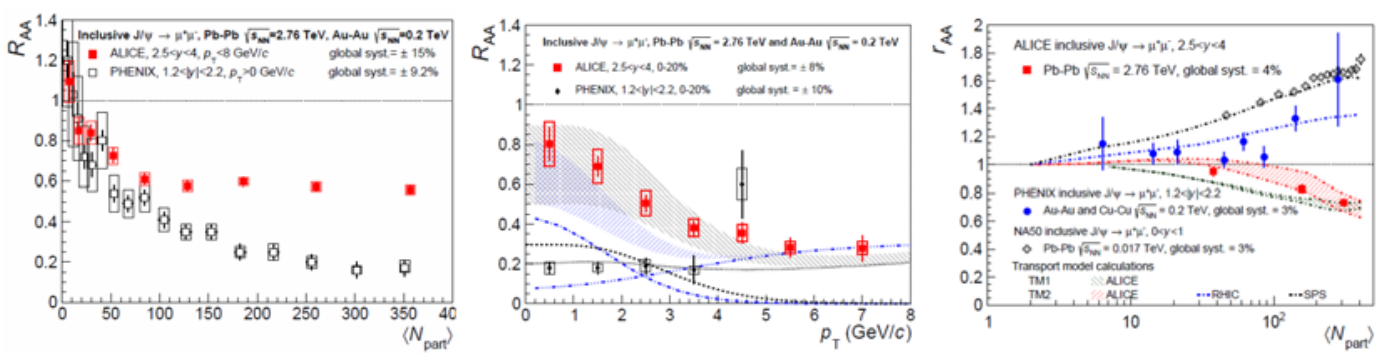

Figure 3. (Left) Nuclear modification factor $R_{A A}$ of inclusive $J / \psi$ as a function of the number of participant nucleons $N_{\text {part }}$ in $\mathrm{PbPb}$ at $2.76 \mathrm{TeV}$ from ALICE [25], compared to the PHENIX data in AuAu at $0.2 \mathrm{TeV}$ [26]. (Center) $R_{A A}$ of inclusive $J / \psi$ as a function of $p_{T}$ for $2.5<y<4$ in central $(0-20 \%) \mathrm{PbPb}$ at $2.76 \mathrm{TeV}$ from ALICE [25], compared to the PHENIX data in AuAu at $0.2 \mathrm{TeV}$ [26]. The calculations from transport models (TM1 [28] and TM2 [5]) are shown for comparison. (Right) Mean $p_{T}$ variable $r_{A A}$ of inclusive $J / \psi$ as a function of $N_{\text {part }}$ from ALICE at 2.76 TeV [25], PHENIX at $0.2 \mathrm{TeV}$ [26, 29, 30], and NA50 at $0.017 \mathrm{TeV}$ [31]. The lines and bands are the calculations from TM1 and TM2.

from various models. The pure energy loss scenario predicts a much stronger $p_{T}$ dependence at forward rapidities. A calculation based on NLO CEM with EPS09 reproduces the $p_{T}$ dependence within uncertainties [24], but a discrepancy still exist between the data and both calculations at the low- $p_{T}$ region. Note that the theoretical uncertainties arise from the uncertainties on EPS09, the charm-quark mass, and the renormalization and factorization scales for the cross section calculation.

\section{Quarkonium production in $\mathrm{PbPb}$}

The centrality dependence of nuclear modification factor $R_{A A}$ for inclusive $J / \psi$ in $\mathrm{PbPb}$ at $2.76 \mathrm{TeV}$ measured by ALICE is shown in the left panel of Fig. 3 [25]. The $J / \psi$ is measured at $p_{T}<8$ $\mathrm{GeV}$ in forward rapidity of $2.5<y<4$ as a function of the number of participant nucleons $N_{\text {part }}$. The suppression is significantly smaller than that observed at lower energy by PHENIX in a similar kinematic range [26]. The suppression is also smaller than that measured at higher $p_{T}(>6.5 \mathrm{TeV})$ at $2.76 \mathrm{TeV}$ from CMS [27]. For $N_{\text {part }}>70$, corresponding to $\sim 50 \%$ most central $\mathrm{PbPb}$ collision, the $J / \psi R_{A A}$ from ALICE is almost constant, which is distinct from the lower energy data.

The $p_{T}$ dependence of $R_{A A}$ for inclusive $J / \psi$ is shown in the middle panel of Fig. 3 for central (0 - $20 \%) \mathrm{PbPb} . R_{A A}$ for inclusive $J / \psi$ shows very little suppression at low $p_{T}$ at LHC, and decreases towards higher $p_{T}$. On contrary the low-energy PHENIX data show no significant $p_{T}$ dependence [26]. The calculations from the two transport models (TM1 [28] and TM2 [5]) are compared to the ALICE data. (The main difference between the two models is the rate equation, controlling the $J / \psi$ dissociation and regeneration processes.) In addition, the primordial component from the initial hard parton scattering and the regenerated component from coalescence of $c$ and $\bar{c}$ are displayed, separately. The primordial components are constant or slightly increase with $p_{T}$, but the regenerated components are concentrated at the low- $p_{T}$ region for both models.

The centrality dependences of the $p_{T}$-width variable $r_{A A}=\left\langle p_{T}^{2}\right\rangle_{A A} /\left\langle p_{T}^{2}\right\rangle_{p p}$ for inclusive $J / \psi$ are compared each other at different energies from ALICE at 2.76 TeV [25], PHENIX at $0.2 \mathrm{TeV}$ [26, 29, 30], and NA50 at $0.017 \mathrm{TeV}$ [31] in the right panel of Fig. 3. A very different $N_{\text {part }}$ dependence is observed: the increase at the lowest energy was attributed to the extra $p_{T}$ kick due to multiple 

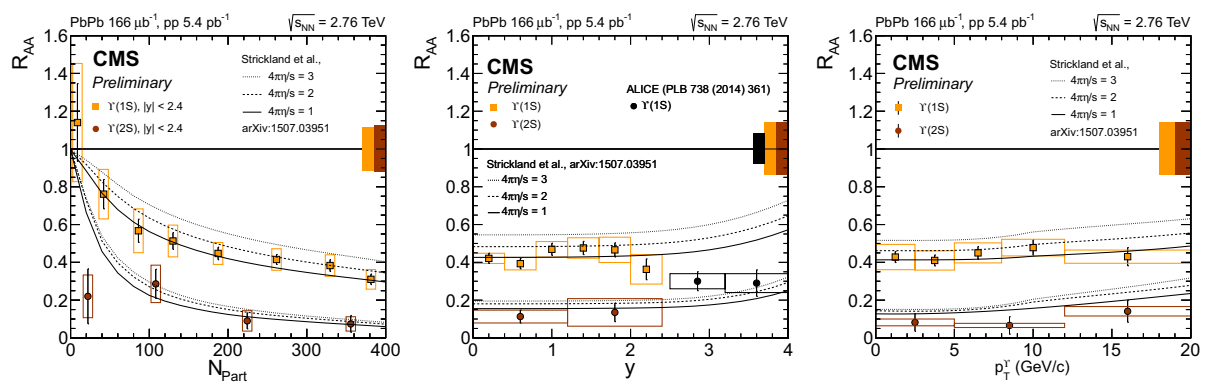

Figure 4. (Left) Nuclear modification factor $R_{A A}$ of $\Upsilon(1 \mathrm{~S})$ and $\Upsilon(2 \mathrm{~S})$ in minimum bias $(0-100 \%) \mathrm{PbPb}$ at $2.76 \mathrm{TeV}$ from CMS as functions of the number of participant nucleons $N_{\text {part }}$ (left), rapidity (center), and $p_{T}$ (right) [33]. The error bars and boxes represent the statistical and systematic, respectively, uncertainties. The boxes at unity show the fully-correlated global uncertainties. The various lines represent the calculations from the anisotropic hydrodynamics with different assumption on the shear-viscosity-to-entropy-density ratio $4 \pi \eta / s$ [35]. For the rapidity distribution, the ALICE data for $\Upsilon(1 \mathrm{~S})$ in $2.5<y<4$ [36] are also plotted for comparison.

scatterings of initial partons (the so-called Cronin effect) [32], and the decrease at the highest energy could be related to the regeneration and the thermalization of charm quarks.

The $R_{A A}$ distributions of $\Upsilon(1 \mathrm{~S})$ and $\Upsilon(2 \mathrm{~S})$ in minimum bias $(0-100 \%) \mathrm{PbPb}$ at $2.76 \mathrm{TeV}$ from CMS [33] are shown in Fig. 4 as functions of $N_{\text {part }}$, rapidity, and $p_{T}$. The strong centrality dependence, previously observed by CMS with larger uncertainties [34], is confirmed with higher precision. The improvement was achieved by $\sim 30 \%$ more statistics for $\Upsilon(1 \mathrm{~S})$ in $\mathrm{PbPb}$ and fully analyzed pp reference events recorded in 2013. On the other hand, the $R_{A A}$ distributions are constant within uncertainties as functions of $p_{T}$ and $y$, and no pronounced dependence on the kinematic variables can be observed. The $R_{A A}$ values, integrated over $p_{T}$ and $y$, are $0.425 \pm 0.029$ (stat) \pm 0.070 (syst) for $\Upsilon(1 \mathrm{~S}$ ) and $0.116 \pm$ 0.028 (stat) \pm 0.022 (syst) for $\Upsilon(2 \mathrm{~S})$. $\Upsilon(3 \mathrm{~S})$ cannot be identified in $\mathrm{PbPb}$, and only upper limit of 0.14 is given at $95 \%$ confidence level.

The $\Upsilon(n S)$ data are compared with the calculations from various models. For example, Fig. 4 shows the comparison with anisotropic hydrodynamics model with different assumptions on the shearviscosity-to-entropy-density ratio $4 \pi \eta / s$ [35]. The centrality dependence of $\Upsilon(1 \mathrm{~S})$ yield agrees better with the model calculation, when smaller shear viscosity $(1 \lesssim 4 \pi \eta / s \lesssim 2)$ is employed. For the rapidity dependence, the model describes the $\Upsilon(1 \mathrm{~S})$ data in $|y|<2.4$ covered by CMS, but fails to reproduce the ALICE data at forward rapidities in $2.5<y<4$ [36]. In the meanwhile, the transport model [4] can also reproduce the centrality dependences of the $\Upsilon(1 S)$ and $\Upsilon(2 S)$ yields only when the regeneration components are included. In particular, the $\Upsilon(2 S)$ yield from semi-central to central $\mathrm{PbPb}$ collisions consists of mostly the regenerated particles according to the transport model.

\section{Summary}

The $J / \psi, \psi(2 \mathrm{~S})$, and $\Upsilon(n \mathrm{~S})$ mesons have been measured in $\mathrm{pp}, \mathrm{pPb}$, and $\mathrm{PbPb}$ collisions by the $\mathrm{LHC}$ experiments. The new pp data at $7 \mathrm{TeV}$ extends the $p_{T}$ reach up to $100 \mathrm{GeV}$, which helps to understand the production mechanism. The $\mathrm{pPb}$ data at $5.02 \mathrm{TeV}$ show less yields in the forward p-going direction than in the backward $\mathrm{Pb}$-going direction at low $p_{T}$, which hints a modification of the parton distribution function in $\mathrm{Pb}$ nucleus. The $J / \psi$ yield in central $\mathrm{PbPb}$ requires the regeneration component. The width of the $p_{T}$ distribution of $J / \psi$ in $\mathrm{PbPb}$ decreases with centrality at LHC energy, which 
can be attributed to the regeneration component. The nuclear modification factors of $\Upsilon(1 \mathrm{~S})$ and $\Upsilon(2 \mathrm{~S})$ do not show any pronounced dependence on the kinematic variables like $p_{T}$ and $y$.

\section{References}

[1] T. Matsui and H. Satz, Phys. Lett. B 178, 416 (1986)

[2] A. Mocsy and P. Petreczky, Phys. Rev. D 77, 014501 (2008)

[3] A. Andronic, P. Braun-Munzinger, K. Redlich, and J. Stachel, J. Phys. G 38, 124081 (2011)

[4] A. Emerick, X. Zhao, and R. Rapp, Eur. Phys. J. A 48, 72 (2012)

[5] K. Zhou, N. Xu, Z. Xu, and P. Zhuang, Phys. Rev. C 89, 054911 (2014)

[6] R. Vogt, Phys. Rev. C 81, 044903 (2010)

[7] CMS Collaboration, Phys. Rev. Lett. 114, 191802 (2015)

[8] ATLAS Collaboration, Nucl. Phys. B 850, 387 (2011)

[9] ATLAS Collaboration, J. High Energy Phys. 09, 079 (2014)

[10] HERA-B Collaboration, Eur. Phys. J. C 49, 545 (2007)

[11] P. Faccioli, V. Knünz, C. Lourenco, J. Seixas, and H. Wöhri, Phys. Lett. B 736, 98 (2014)

[12] CMS Collaboration, arXiv: 1501.07750

[13] CMS Collaboration, Phys. Lett. B 727, 101 (2013)

[14] B. Gong, L.-P. Wan, J.-X.Wang, and H.-F. Zhang, Phys. Rev. Lett. 112, 032001 (2014)

[15] CMS Collaboration, J. High Energy Phys. 04, 103 (2014)

[16] ALICE Collaboration, Phys. Lett. B 712, 165 (2012)

[17] H. Abramowicz, et al., arXiv:1306.5413

[18] ALICE Collaboration, J. High Energy Phys. 02, 073 (2014)

[19] J. L. Albacete, et al., Int. J. Mod. Phys. E 22, 1330007 (2013)

[20] F. Arleo and S. Peigne, J. High Energy Phys. 03, 122 (2013)

[21] H. Fujii and K. Watanabe, Nucl. Phys. A 915, 1 (2013)

[22] CMS Collaboration, "Measurement of prompt and non-prompt $J / \psi$ production in $\mathrm{pPb}$ collisions at $\sqrt{s_{N N}}=5.02 \mathrm{TeV}$ TeV", CMS PAS HIN-14-009

[23] ALICE Collaboration, J. High Energy Phys. 06, 055 (2015)

[24] F. Arleo, R. Kolevatov, S. Peigne and M. Rustamova, J. High Energy Phys. 05, 155 (2013)

[25] ALICE Collaboration, arXiv:1506.08804

[26] PHENIX Collaboration, Phys. Rev. C 84, 054912 (2011)

[27] CMS Collaboration, "Prompt and non-prompt $J / \psi R_{A A}$ with $150 \mu \mathrm{b}^{-1}$ integrated $\mathrm{PbPb}$ luminosity at $\sqrt{s_{N N}}=2.76$ TeV", CMS PAS HIN-12-014

[28] X. Zhao and R. Rapp, Nucl. Phys. A 859, 114 (2011)

[29] PHENIX Collaboration, Phys. Rev. Lett. 98, 232002 (2007)

[30] PHENIX Collaboration, Phys. Rev. Lett. 101, 122301 (2008)

[31] NA50 Collaboration, Phys. Lett. B 499, 85 (2001)

[32] J. Cronin, H. J. Frisch, M. Shochet, J. Boymond, and R. Mermod et al., Phys. Rev. D 11, 3105 (1975)

[33] CMS Collaboration, "Suppression of $\Upsilon(1 \mathrm{~S}), \Upsilon(2 \mathrm{~S})$ and $\Upsilon(3 \mathrm{~S})$ in $\mathrm{PbPb}$ collisions at $\sqrt{s_{N N}}=2.76$ TeV TeV”, CMS PAS HIN-15-001

[34] CMS Collaboration, Phys. Rev. Lett. 109, 222301 (2012)

[35] B. Krouppa, R. Ryblewski, and M. Strickland, arXiv:1507.03951

[36] ALICE Collaboration, Phys. Lett. B 738, 361 (2014) 